\title{
Monitoring cardiac fibrosis: a technical challenge
}

\author{
S. de Jong • T. A. B. van Veen • J. M. T. de Bakker • \\ H. V. M. van Rijen
}

Published online: 9 December 2011

(C) The Author(s) 2011. This article is published with open access at Springerlink.com

\begin{abstract}
The heart contains a collagen network that contributes to the contractility of the heart and provides cardiac strength. In cardiac diseases, an increase in collagen deposition is often observed. This fibrosis formation causes systolic and diastolic dysfunction, and plays a major role in the arrythmogenic substrate. Therefore, accurate detection of cardiac fibrosis and its progression is of clinical importance with regard to diagnostics and therapy for patients with cardiac disease. To evaluate cardiac collagen deposition, both invasive and non-invasive techniques are used. In this review the different techniques that are currently used in clinical and experimental setting are summarised, and the advantages and disadvantages of these techniques are discussed.
\end{abstract}

Keywords Collagen $\cdot$ Fibrosis $\cdot$ Detection techniques

\section{Introduction}

Cardiac tissue consists mainly of cardiomyoctyes, which are surrounded by extracellular matrix. The major component of the extracellular matrix is collagen. Different types of collagen exist, and the fibrillar collagen types I and III are most abundant in the myocardium. These collagen fibres form a collagen network that provides cardiac strength during contractile

S. de Jong $(\varangle) \cdot$ T. A. B. van Veen · J. M. T. de Bakker •

H. V. M. van Rijen

Department of Medical Physiology,

University Medical Center Utrecht,

Yalelaan 50,

3584 CM Utrecht, the Netherlands

e-mail: s.dejong-9@umcutrecht.nl

J. M. T. de Bakker

Interuniversity Cardiology Institute of the Netherlands,

Utrecht, the Netherlands forces, and collagen fibres play a role in myocyte - myocyte connections [1].

In virtually all cardiac diseases, an increase in collagen deposition is observed, hence fibrosis. Two types of fibrosis are distinguished: replacement and reactive fibrosis. When cardiac cell death occurs, these cells are replaced by collagen, called reparative fibrosis. However, fibrosis may also develop without cell loss, e.g. due to increased mechanical load. This type of collagen deposition is called reactive fibrosis and is observed as patchy fibrosis in the myocardium or large collagen strands in between the cardiomyocytes (interstitial fibrosis). Both types of fibrosis lead to diastolic and systolic dysfunction [2], and reactive fibrosis leads to an increased susceptibility for cardiac arrhythmias [3].

For an appropriate prognosis and therapy of cardiac patients, knowledge about collagen content and the progression of fibrosis formation is important. Various invasive and non-invasive techniques are currently available to detect collagen and new methods are still being developed. This review focuses on detection techniques that are currently used for cardiac collagen monitoring, both in the clinical and experimental setting. Advantages and disadvantages of the various techniques are discussed.

\section{Collagen turnover}

Collagen is not static, but is continuously synthesised and degraded. Procollagen, the precursor of collagen, is synthesised by fibroblasts as a triple helix molecule. Once procollagen is transported from the fibroblast into the intercellular space, propeptides at the amino (N)- and carboxy (C)-terminus of the procollagen are cleaved. The mature collagen will form larger collagen fibrils that become part of the collagen network [4] (Fig. 1). Degradation of collagen is provided by matrix 
Fig. 1 Schematic overview from the synthesis of procollagen to embedding of the collagen into the network 1 . Three procollagen strands, synthesised by the fibroblast, form procollagen with its triple helical structure. 2. Procollagen is transported into the extracellular matrix and the propeptides are cleaved. 3. The mature collagen forms larger fibrils and cross-links and is embedded in the collagen network of the heart

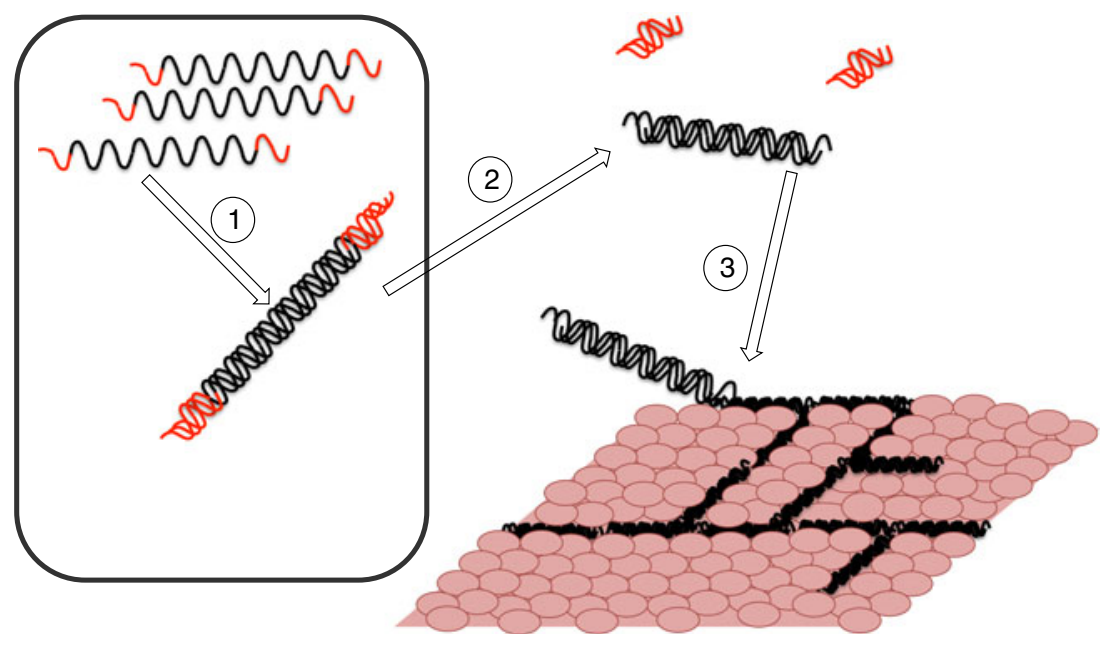

metalloproteases (MMPs) that can be regulated by tissue inhibitors of metalloproteases (TIMPs) [5].

Collagen is synthesised at a rate of $5 \%$ per day. A similar amount is degraded in the non-growing heart [5]. In diseased myocardium, the equilibrium between collagen synthesis and breakdown is disturbed, resulting in collagen deposition.

\section{Invasive techniques to detect collagen}

Cardiac tissue biopsies harvested during surgery or with catheters can be used to investigate the structural changes in the myocardium. Although taking biopsies is unfavourable for the patient and not without risk, the collection of tissue is still widely used and provides detailed information about structural and electrical remodelling of the heart. The disadvantage of a biopsy is that analyses of this biopsy only provide information on a very localised area of the heart.

\section{Histology}

For a histological examination of the collagen content, several routinely used and cheap dye stains can be used. For this purpose tissue is frozen or embedded in paraffin and cut in sections with a thickness of about 4-10 $\mu \mathrm{m}$. Masson's Trichrome and Picriosirius Red are the most frequently used stains for collagen. Masson's Trichrome is based on three dyes, of which Light Green or Aniline Blue are commonly used to colour the collagen [6]. In the Picrosirius Red stain, the Picrosirius Red, which is extremely acid, binds to collagen [7]. Also phosphomolybdic acid, one of the dyes used in Masson's Trichrome, is able to bind collagen. Subsequently, Light Green or Aniline Blue interacts [6]. Thus, binding of these dyes is based on base-acid interactions, and may therefore not be specific for collagen.
Since mature collagen is birefringent, and Picrosirius Red can enhance the birefringency of collagen, stained collagen fibres can be visualised better and in more detail using polarised light. Whittaker et al. showed that analysis of cardiac collagen with polarised light provides more insight into the structure and composition of the collagen network, as compared to Picrosirius Red or Masson's Trichrome staining [8].

To be more collagen specific, fluorescent labelled antibodies can be used. Commercially available antibodies can be used for specific protein investigations with Western blot or immunohistology. This method is less frequently used, mainly because antibodies are more expensive than dye stains and immunohistology is more time-consuming. Moreover, when using immunofluorescent microscopy, autofluorescence of the tissue may interfere with a quantitative analysis.

\section{CNA35}

CNA35 is a truncated bacterial protein that binds to collagen. Krahn et al. have shown that fluorescent labels can easily be attached to CNA35 and that such fluorescent probes are still highly specific for collagen. The authors state that fluorescent labelled CNA35 provides the following advantages when compared with antibodies: 1) the probes have a high affinity to bind to collagen, but binding is reversible $\left(\mathrm{K}_{\mathrm{d}}=10^{-7}-10^{-6} \mathrm{M}\right)$, which is a prerequisite for imaging of collagen in viable tissue; and 2) labelled CNA35 is much smaller than antibodies, which might be beneficial for an optimal penetration of tissue [9]. Moreover, the fluorescent label at CNA35 can be replaced by a MRI tagged label such as gadopentetate dimeglumine (Gd-DTPA; a gadolinium-based contrast agent), which would allow specific collagen detection by MRI. Although in vivo studies with mice showed no fluorescent staining in the myocardium after intravenous injection of CNA35 [10], preliminary 
experiments ex vivo show promising results for cardiac collagen detection with CNA35. In collagen-rich heart sections and in collagen-rich hearts where labelled CNA35 is perfused via a Langendorff setup, the fluorescent signal is detected in the sections and coincides with a Picrosirius Red staining on serial sections (Fig. 2; personal communication from R. Hermans, MSc., Prof. Dr. M. van Zandvoort, and Prof. Dr. F. Prinzen, Maastricht University). Further research is required to investigate whether labelled CNA35 can be used in monitoring cardiac collagen in vivo.

\section{Hydroxyproline assay}

The hydroxylation (introduction of a hydroxyl-group into an organic compound) of proline within the collagen molecule results in hydroxyproline. This modified amino acid stabilises the triple helical structure in mature collagen [11]. Since hydroxyproline is highly specific for collagen, hydroxyproline measurements accurately reflect the amount of collagen in the tissue. Commercial kits are available to determine hydroxyproline. Collagen contains $14 \%$ hydroxyproline, hence the actual amount of collagen can easily be calculated. However, for this analysis homogenised tissue samples are required and will therefore provide no information on potential heterogeneity of deposition, which might be demanded in e.g. ablation surgery. Despite this disadvantage, the hydroxyproline assay is still one of the most reliable techniques to assess total collagen tissue levels.

\section{Sircol assay}

The commercially available Sircol assay is based on the binding of Picrosirius Red to collagen in homogenised samples. In this assay tissue is homogenised with pepsin, and only newly formed collagen is measured. This is because only procollagen can be dissolved in pepsin, whereas crosslinked collagen will remain insoluble [12]. The analysis is easy to perform, but as with the hydroxyproline assay, the assay does not provide any information about the sitespecific localisation of the collagen either.

\section{Non-invasive techniques to detect collagen}

\section{Circulating biomarkers in the blood}

When the C- and N-terminus of procollagen are cleaved from procollagen, they are released into the bloodstream. The N-terminus of procollagen III (PIIINP) and the C-and $\mathrm{N}$-terminus of procollagen I (PICP and PINP respectively) can be detected in blood samples [13]. Circulating biomarkers that reflect breakdown of the collagen are MMPs, TIMPs, and one of the breakdown products of collagen I, ICTP [13]. Several studies have investigated the levels of these markers for various cardiac diseases, but results are frequently conflicting [14].

The biomarkers mentioned before can be determined in blood samples with commercially available assays. Advantages of measuring biomarkers with these kits are that the analysis is non-invasive, cheap, and easily applicable in the clinical setting. However, the quantitative analysis of cardiac fibrosis by these biomarkers is not yet validated [14].

Another limitation of these circulatory biomarkers is that the circulating peptides are not cardiac-specific. As collagen I is the most prominent collagen type in all tissues, the biomarker levels of collagen I will reflect the collagen type I turnover in the entire body. There are indications that the described biomarkers also reflect cardiac collagen: 1) some biomarkers have a gradient from coronary sinus blood to peripheral blood, and

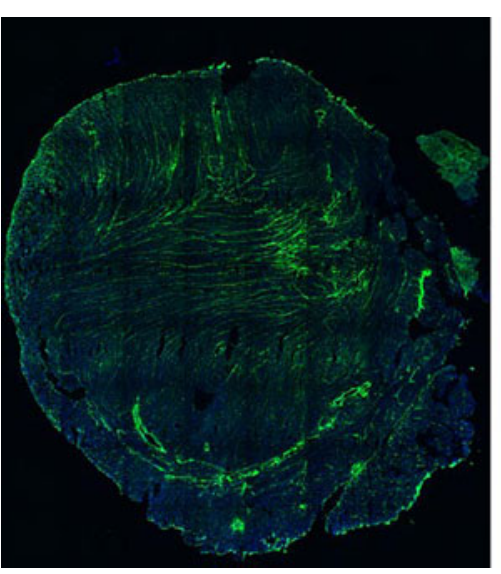

Fig. 2 Collagen detection with CNA35. The left panel shows a coronal section of a mouse heart containing interstitial fibrosis that is detected by CNA35 labelled with the fluorescent probe FITC. Green $=$ collagen, blue $=$ nuclei. The right panel shows a serial

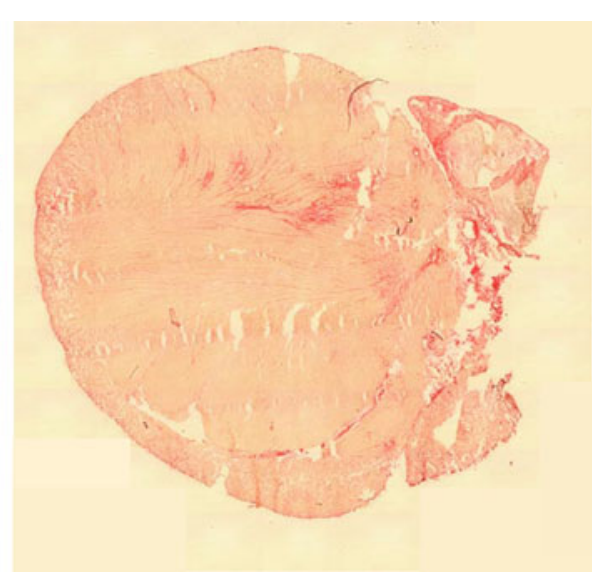

section of the mouse heart stained with Picrosirius Red, yellow = viable myocardium, red = collagen. CNA35-stained picture kindly provided by Prof. Dr. Prinzen and Prof. Dr. van Zandvoort, Maastricht University 
2) levels in patients with cardiac disease differ from healthy controls (reviewed in De Jong et al. [14]). However, it would be of great advantage to look for circulating biomarkers that specifically predict cardiac collagen. In this respect, microRNAs (miRNAs) might be of particular interest. MiRNAs are post-transcriptional regulators (short RNA strands) of a number of genes. It has been shown that miRNAs such as miR-29, miR-30, and miR-21 play a specific role in collagen formation in the myocardium, as nicely reviewed by Bauersachs [15]. Recent studies showed that miRNA levels detected in blood samples were significantly changed in patients with acute myocardial infarction [16] or with heart failure [17] compared with healthy controls. Whether miRNAs specifically involved in cardiac fibrosis formation are also detectable in blood is currently unknown, but might be of particular interest for specific collagen biomarkers.

\section{MRI}

Late gadolinium enhanced (LGE) MRI is frequently used in clinics nowadays to assess cardiac scar tissue. Myocardial scarring can be detected using MRI after administration of a gadolinium-based contrast agent. Due to differences in washout kinetics of scarred and viable tissue, the contrast agent remains longer in scarred than in viable tissue [18]. Although this technique has greatly improved detection of myocardial fibrosis in the clinical setting, the method has some drawbacks. In the first place, it represents an indirect method for collagen detection of collagen deposition. Moreover, currently only large areas of scarring can be detected while small patches of fibrosis and increased interstitial fibrosis are of particular interest in arrhythmia susceptibility and systolic dysfunction.

Extensive research is still being performed to improve fibrosis detection by imaging techniques. A recent study of Messroghli et al. showed that patchy and interstitial cardiac fibrosis in a hypertrophic rat model can be determined with MRI T1 mapping based on a gadolinium-based contrast agent. The authors showed a moderate though significant correlation between myocardial extracellular volume measured by Gd-DTPA based T1 mapping and histological analysis of cardiac fibrosis [19].

We have recently shown that ultrashort echo time (UTE) MRI is another technique that can be used for detection of myocardial fibrosis. UTE MRI can detect tissues with very fast signal decay, such as tendons and bone. These compact tissues are collagen-rich, and are thought to be detected by UTE MRI since collagen has very fast signal decay. To investigate whether cardiac collagen could be detected with UTE MRI as well, we used a rat myocardial infarction model. This study showed that UTE MRI is able to detect myocardial fibrosis after infarction without the use of any contrast agents [20].
Imaging techniques are favourable for the detection of collagen since these techniques are non-invasive. However, a major drawback of the use of imaging techniques for detection of fibrosis is that quantification of the hyperenhanced signals on MR images is not yet standardised. Flett et al. compared several quantification techniques for LGE MRI images and showed that substantial differences exist in the amount of fibrosis measured by the different techniques. The full width half max (FWHM) technique appeared the most reliable and reproducible quantification technique [18].

\section{Concluding remarks}

Cardiac collagen plays an important role in cardiac disease, because it may affect contractility and arrhythmogenicity. Thus, detection of collagen in patients with heart disease is of utmost importance. In this review several techniques that are used or developed for collagen detection in the heart are discussed. Both invasive and non-invasive techniques are available. Histological and biochemical assessment of collagen in cardiac tissue is still the most accurate method and easy to quantify, especially the hydroxyproline assay, but size-limited cardiac biopsies are required for these analyses. Whereas the non-invasive techniques such as MRI and circulating biomarkers are of great interest, quantitative analysis of these techniques are not yet standardised and validated respectively in patients.

Acknowledgement This research was performed within the framework of CTMM, the Center for Translational Molecular Medicine (www.ctmm.nl), project COHFAR (grant 01C-203), and supported by the Netherlands Heart Foundation.

Open Access This article is distributed under the terms of the Creative Commons Attribution Noncommercial License which permits any noncommercial use, distribution, and reproduction in any medium, provided the original author(s) and source are credited.

\section{References}

1. Eghbali M, Weber KT. Collagen and the myocardium: fibrillar structure, biosynthesis and degradation in relation to hypertrophy and its regression. Mol Cell Biochem. 1990;96(1):1-14.

2. Janicki JS, Brower GL. The role of myocardial fibrillar collagen in ventricular remodeling and function. J Card Fail. 2002;8(6 Suppl): S319-25.

3. de Jong S, van Veen TA, van Rijen HV, et al. Fibrosis and cardiac arrhythmias. J Cardiovasc Pharmacol. 2011;57(6):630-8.

4. van der Rest M, Garrone R. Collagen family of proteins. FASEB J. 1991;5(13):2814-23.

5. Weber KT, Sun Y, Katwa LC, et al. Connective tissue and repair in the heart. Potential regulatory mechanisms. Ann N Y Acad Sci. 1995;752:286-99. 
6. Puchtler H, Isler H. The effect of phosphomolybdic acid on the stainability of connective tissues by various dyes. J Histochem Cytochem. 1958;6(4):265-70.

7. Sweat F, Puchtler H, Rosenthal SI. Sirius red F3ba as a stain for connective tissue. Arch Pathol. 1964;78:69-72.

8. Whittaker P, Kloner RA, Boughner DR, et al. Quantitative assessment of myocardial collagen with picrosirius red staining and circularly polarized light. Basic Res Cardiol. 1994;89(5):397-410.

9. Krahn KN, Bouten CV, van Tuijl S, et al. Fluorescently labeled collagen binding proteins allow specific visualization of collagen in tissues and live cell culture. Anal Biochem. 2006;350(2):17785.

10. Megens RT, Oude Egbrink MG, Cleutjens JP, et al. Imaging collagen in intact viable healthy and atherosclerotic arteries using fluorescently labeled CNA35 and two-photon laser scanning microscopy. Mol Imaging. 2007;6(4):247-60.

11. Berg RA, Prockop DJ. The thermal transition of a nonhydroxylated form of collagen. Evidence for a role for hydroxyproline in stabilizing the triple-helix of collagen. Biochem Biophys Res Commun. 1973;52(1):115-20.

12. Miller EJ. Structural studies on cartilage collagen employing limited cleavage and solubilization with pepsin. Biochemistry. 1972;11(26):4903-9.
13. Risteli J, Risteli L. Collagen metabolites in body fluids. 2nd ed. New York: Wiley-Liss; 2002.

14. de Jong S, van Veen TA, de Bakker JM, et al. Biomarkers of myocardial fibrosis. J Cardiovasc Pharmacol. 2011;57(5):522-35.

15. Bauersachs J. Regulation of myocardial fibrosis by MicroRNAs. J Cardiovasc Pharmacol. 2010;56(5):454-9.

16. Wang GK, Zhu JQ, Zhang JT, et al. Circulating microRNA: a novel potential biomarker for early diagnosis of acute myocardial infarction in humans. Eur Heart J. 2010;31(6):659-66.

17. Fukushima $Y$, Nakanishi $M$, Nonogi $H$, et al. Assessment of plasma miRNAs in congestive heart failure. Circ J. 2011;75 (2):336-40.

18. Flett AS, Hasleton J, Cook C, et al. Evaluation of techniques for the quantification of myocardial scar of differing etiology using cardiac magnetic resonance. JACC Cardiovasc Imaging. 2011;4 (2):150-6.

19. Messroghli D, Nordmeyer S, Dietrich T, et al. Assessment of diffuse myocardial fibrosis in rats using small animal look-locker inversion recovery (SALLI) T1 mapping. Circ Cardiovasc Imaging. 2011. doi:10.1161/CIRCIMAGING.111.966796.

20. de Jong S, Zwanenburg JJ, Visser F, et al. Direct detection of myocardial fibrosis by MRI. J Mol Cell Cardiol. 2011. doi:10.1016/j. yjmcc.2011.08.024. 